\title{
Differential games with continuous, switching and impulse controls
}

\author{
A.J. Shaiju ${ }^{\mathrm{a}, 1}$, Sheetal Dharmatti ${ }^{\mathrm{b}, *, 2}$ \\ ${ }^{a}$ TIFR Centre, IISc Campus, Bangalore 560012, India \\ ${ }^{\mathrm{b}}$ Department of Mathematics, Indian Institute of Science, Bangalore 560 012, India
}

\begin{abstract}
A two-person zero-sum differential game of infinite duration with discounted payoff involving hybrid controls is studied. The minimizing player is allowed to take continuous, switching, and impulse controls whereas the maximizing player is allowed to take continuous and switching controls. By taking strategies in the sense of Elliott-Kalton, we prove the existence of value and characterize it as the unique viscosity solution of the associated system of quasi-variational inequalities.
\end{abstract}

Keywords: Differential games; Viscosity solutions; Strategy; Mixed controls; Value

\section{Introduction and preliminaries}

The study of differential games with Elliott-Kalton strategies in the viscosity solution framework is initiated by Evans and Souganidis [2] where both players are allowed to take continuous controls. Differential games where both players use switching controls are studied by Yong [3,4]. In [5], differential games involving impulse controls are considered; one player is using continuous controls whereas the other uses impulse control. In the final section of [5], the author mentions that by applying the ideas and techniques used in the previous sections one can study differential games where one player uses continuous,

\footnotetext{
* Corresponding author. Tel.: +91 08022932265 ; fax: +910802360 0851.

E-mail addresses: shaiju@math.tifrbng.res.in (A.J. Shaiju), sheetal@math.iisc.ernet.in (S. Dharmatti).

${ }^{1}$ The financial support to A.J. Shaiju from NBHM is gratefully acknowledged.

${ }^{2}$ The financial support to S. Dharmatti from UGC is gratefully acknowledged.
} 
switching, and impulse controls and the other player uses continuous and switching controls. The uniqueness result for the associated system of quasi-variational inequalities (SQVI for short) with bilateral constraints is established under suitable nonzero loop switching-cost condition and cheaper switching condition.

In this paper, we study a two-person zero-sum differential game where the minimizer (Player 2) uses three types of controls: continuous, switching and impulse. The maximizer (Player 1) uses continuous and switching controls. We first prove dynamic programming principle (DPP for short) for this problem. Using this, we prove that the lower and upper value functions satisfy the associated SQVI in the viscosity sense. Finally, we establish the existence of the value by proving a uniqueness theorem for SQVI. We obtain our results without any assumption like nonzero loop switching-cost condition and/or cheaper switching-cost condition on the cost functions. This will be further explained in the concluding section. Hence, we prove the existence of the value under Issacs'-type condition alone.

The paper is organized as follows. We set up necessary notations and assumptions in the rest of this section. The statement of the main result is also given at the end of this introductory section. The DPP is proved in Section 2. Here, we also show that the lower/upper value function is a viscosity solution of SQVI. Section 3 is devoted to the proof of the main uniqueness result for SQVI and the existence of value. The paper ends in Section 4 with some concluding remarks.

We first describe the notations and basic assumptions. The state space is a Euclidean space $\mathbb{R}^{d}$. The continuous control set for Player $i, i=1,2$, is $U^{i}$, a compact metric space. The set $D^{i}=\left\{d_{1}^{i}, \ldots, d_{m_{i}}^{i}\right\} ; i=1,2$, is the switching control set for Player $i$. The impulse control set for the Player 2 is $K$, a compact subset of the state space $\mathbb{R}^{d}$. The space of all $U^{i}$-valued measurable maps on $[0, \infty)$ is continuous control space for Player $i$ and is denoted by $\mathscr{U}^{i}$. By $\mathscr{U}^{i}[0, t]$ we mean the space of all $U^{i}$ valued measurable maps on $[0, t]$. The switching control space $\mathscr{D}^{i}$ and the impulse control space $\mathscr{K}$ are defined as follows:

$$
\begin{aligned}
& \mathscr{D}^{i}=\left\{d^{i}(\cdot)=\sum_{j \geqslant 1} d_{j-1}^{i} \chi_{\left[\theta_{j-1}^{i}, \theta_{j}^{i}\right)}(\cdot): d_{j}^{i} \in D^{i},\left(\theta_{j}^{i}\right) \subset[0, \infty], \theta_{0}^{i}=0,\left(\theta_{j}^{i}\right) \uparrow \infty,\right. \\
&\left.d_{j-1}^{i} \neq d_{j}^{i} \text { if } \theta_{j}^{i}<\infty\right\}, \\
& \mathscr{K}=\left\{\xi(\cdot)=\sum_{j \geqslant 0} \xi_{j} \chi_{\left[\tau_{j}, \infty\right]}(\cdot): \xi_{j} \in K,\left(\tau_{j}\right) \subset[0, \infty],\left(\tau_{j}\right) \uparrow \infty\right\} .
\end{aligned}
$$

An impulse control $\xi(\cdot)=\sum_{j \geqslant 0} \xi_{j} \chi_{\left[\tau_{j}, \infty\right]}(\cdot)$, consists of the impulse times $\tau_{j}$ 's and impulse vectors $\xi_{j}$ 's. We use the notation $(\xi)_{1, j}$ to denote $\tau_{j}$ and $(\xi)_{2, j}$ to denote $\xi_{j}$. Similarly for switching controls $d^{1}(\cdot)$ and $d^{2}(\cdot)$ we write,

$$
\begin{aligned}
& \left(d^{1}\right)_{1, j}=\theta_{j}^{1} \quad \text { and } \quad\left(d^{1}\right)_{2, j}=d_{j}^{1}, \\
& \left(d^{2}\right)_{1, j}=\theta_{j}^{2} \quad \text { and } \quad\left(d^{2}\right)_{2, j}=d_{j}^{2} .
\end{aligned}
$$


Now we describe the dynamics and cost functions involved in the game. To this end, let $\mathscr{C}^{1}=\mathscr{U}^{1} \times \mathscr{D}^{1}$ and $\mathscr{C}^{2}=\mathscr{U}^{2} \times \mathscr{D}^{2} \times \mathscr{K}$. For $\left(u^{1}(\cdot), d^{1}(\cdot)\right) \in \mathscr{C}^{1}$ and $\left(u^{2}(\cdot), d^{2}(\cdot), \xi(\cdot)\right) \in \mathscr{C}^{2}$, the corresponding state $y_{x}(\cdot)$ is governed by the following hybrid controlled system:

$$
\dot{y}_{x}(t)=f\left(y_{x}(t), u^{1}(t), d^{1}(t), u^{2}(t), d^{2}(t)\right)+\dot{\xi}(t), \quad y_{x}(0-)=x,
$$

where $f: \mathbb{R}^{d} \times U^{1} \times D^{1} \times U^{2} \times D^{2} \rightarrow \mathbb{R}^{d}$.

We assume that

(A1) The function $f$ is bounded, continuous and for all $x, y \in \mathbb{R}^{d}, d^{i} \in D^{i}, u^{i} \in U^{i}$,

$$
\left\|f\left(x, u^{1}, d^{1}, u^{2}, d^{2}\right)-f\left(y, u^{1}, d^{1}, u^{2}, d^{2}\right)\right\| \leqslant L\|x-y\| .
$$

Note that under assumption (A1), for each $x \in \mathbb{R}^{d}, d^{i}(\cdot) \in \mathscr{D}^{i}, u^{i}(\cdot) \in \mathscr{U}^{i}$, and $\xi(\cdot) \in \mathscr{K}$ there is a unique solution $y_{x}(\cdot)$ of (1.1).

Let $k: \mathbb{R}^{d} \times U^{1} \times D^{1} \times U^{2} \times D^{2} \rightarrow \mathbb{R}$ be the running cost function, $c^{i}: D^{i} \times D^{i} \rightarrow \mathbb{R}$ the switching cost functions, and $l: K \rightarrow \mathbb{R}$ the impulse cost function.

We assume that

(A2) the cost functions $k, c^{i}, l$ are nonnegative, bounded, continuous, and for all $x, y \in \mathbb{R}^{d}$, $d^{i} \in D^{i}, u^{i} \in U^{i}, \xi_{0}, \xi_{1} \in K$,

$$
\begin{aligned}
& \left|k\left(x, u^{1}, d^{1}, u^{2}, d^{2}\right)-k\left(y, u^{1}, d^{1}, u^{2}, d^{2}\right)\right| \leqslant L\|x-y\|, \\
& l\left(\xi_{0}+\xi_{1}\right)<l\left(\xi_{0}\right)+l\left(\xi_{1}\right) \quad \forall \xi_{0}, \xi_{1} \in K, \\
& \inf _{d_{1}^{i} \neq d_{2}^{i}} c^{i}\left(d_{1}^{i}, d_{2}^{i}\right)=: c_{0}^{i}>0 .
\end{aligned}
$$

Let $\lambda>0$ be the discount parameter. The total discounted cost functional $J_{x}: \mathscr{C}^{1} \times \mathscr{C}^{2} \rightarrow$ $\mathbb{R}$ is given by

$$
\begin{aligned}
J_{x} & {\left[u^{1}(\cdot), d^{1}(\cdot), u^{2}(\cdot), d^{2}(\cdot), \xi(\cdot)\right] } \\
= & \int_{0}^{\infty} \mathrm{e}^{-\lambda t} k\left(y_{x}(t), u^{1}(t), d^{1}(t), u^{2}(t), d^{2}(t)\right) \mathrm{d} t \\
& -\sum_{j \geqslant 0} \mathrm{e}^{-\lambda \theta_{j}^{1}} c^{1}\left(d_{j-1}^{1}, d_{j}^{1}\right)+\sum_{j \geqslant 0} \mathrm{e}^{-\lambda \theta_{j}^{2}} c^{2}\left(d_{j-1}^{2}, d_{j}^{2}\right)+\sum_{j \geqslant 1} \mathrm{e}^{-\lambda \tau_{j}} l\left(\xi_{j}\right) .
\end{aligned}
$$

We next define the strategies and value functions for Players 1 and 2 in the Elliott-Kalton framework. The strategy set $\Gamma$ for Player 1 is the collection of all nonanticipating maps $\alpha$ from $\mathscr{C}^{2}$ to $\mathscr{C}^{1}$. The strategy set $\Delta$ for Player 2 is the collection of all nonanticipating maps $\beta$ from $\mathscr{C}^{1}$ to $\mathscr{C}^{2}$.

For a strategy $\beta$ of Player 2 if $\beta\left(u^{1}(\cdot), d^{1}(\cdot)\right)=\left(u^{2}(\cdot), d^{2}(\cdot), \xi(\cdot)\right)$, then we write $\Pi_{1}$ $\beta\left(u^{1}(\cdot), d^{1}(\cdot)\right)=u^{2}(\cdot), \Pi_{2} \beta\left(u^{1}(\cdot), d^{1}(\cdot)\right)=d^{2}(\cdot)$ and $\Pi_{3} \beta\left(u^{1}(\cdot), d^{1}(\cdot)\right)=\xi(\cdot)$. That is, $\Pi_{i}$ is the projection on the $i$ th component of the map $\beta$. Similar notations we use for $\alpha\left(u^{2}(\cdot), d^{2}(\cdot), \xi(\cdot)\right)$ as well.

Let $\mathscr{D}^{i, d^{i}}$ denote the set of all switching controls for Player $i$ starting at $d^{i}$. Then we define sets $\mathscr{C}^{1, d^{1}}=\mathscr{U}^{1} \times \mathscr{D}^{1, d^{1}}$ and $\mathscr{C}^{2, d^{2}}=\mathscr{U}^{2} \times \mathscr{D}^{2, d^{2}} \times \mathscr{K}$. The collection of all $\beta \in \Delta$ such that $\Pi_{2} \beta(0-)=d^{2}$ is denoted by $\Delta^{d^{2}}$ and the collection of all $\alpha \in \Gamma$ such that $\Pi_{2} \alpha(0-)=d^{1}$ 
is denoted by $\Gamma^{d^{1}}$. Let $\Gamma_{0}^{d^{1}}$ denote the set of all $\alpha \in \Gamma^{d^{1}}$ with $\left(\Pi_{2} \alpha\right)_{1,1}=\infty$, and $\Delta_{0}^{d^{2}}$ the set of all $\beta \in \Delta^{d^{2}}$ with $\left(\Pi_{2} \beta\right)_{1,1}=\left(\Pi_{3} \beta\right)_{1,1}=\infty$. An impulse control $\xi(\cdot)$ with $(\xi)_{1,1}=\infty$ is denoted by $\xi^{\infty}(\cdot)$.

The upper and lower value functions are defined, respectively, as follows:

$$
\begin{aligned}
& V_{+}^{d^{1}, d^{2}}(x)=\sup _{\alpha \in \Gamma^{d^{1}}} \inf _{\mathscr{C}^{2}, d^{2}} J_{x}^{d^{1}, d^{2}}\left[\alpha\left(u^{2}(\cdot), d^{2}(\cdot), \xi(\cdot)\right), u^{2}(\cdot), d^{2}(\cdot), \xi(\cdot)\right], \\
& V_{-}^{d^{1}, d^{2}}(x)=\inf _{\beta \in \Delta^{d^{2}}} \sup _{\mathscr{C}^{1, d^{1}}} J_{x}^{d^{1}, d^{2}}\left[u^{1}(\cdot), d^{1}(\cdot), \beta\left(u^{1}(\cdot), d^{1}(\cdot)\right)\right],
\end{aligned}
$$

where $J_{x}^{d^{1}, d^{2}}$ is the restriction of the cost functional $J_{x}$ to $\mathscr{C}^{1, d^{1}} \times \mathscr{C}^{2, d^{2}}$.

Let $V_{+}=\left\{V_{+}^{d^{1}, d^{2}}:\left(d^{1}, d^{2}\right) \in D^{1} \times D^{2}\right\}$ and $V_{-}=\left\{V_{-}^{d^{1}, d^{2}}:\left(d^{1}, d^{2}\right) \in D^{1} \times D^{2}\right\}$. If $V_{+} \equiv V_{-} \equiv V$, then we say that the differential game has a value and $V$ is referred to as the value function.

Since all cost functions involved are bounded, value functions are also bounded. In view of (A1) and (A2), the proof for uniform continuity of $V_{+}$and $V_{-}$is standard. Hence, both $V_{+}$and $V_{-}$belong to $B U C\left(\mathbb{R}^{d} ; \mathbb{R}^{m_{1} \times m_{2}}\right)$.

For $x, p \in \mathbb{R}^{d}$, let

$$
\begin{aligned}
& H_{-}^{d^{1}, d^{2}}(x, p)=\max _{u^{2} \in U^{2}} \min _{u^{1} \in U^{1}}\left[\left\langle-p, f\left(x, u^{1}, d^{1}, u^{2}, d^{2}\right)\right\rangle-k\left(x, u^{1}, d^{1}, u^{2}, d^{2}\right)\right], \\
& H_{+}^{d^{1}, d^{2}}(x, p)=\min _{u^{1} \in U^{1}} \max _{u^{2} \in U^{2}}\left[\left\langle-p, f\left(x, u^{1}, d^{1}, u^{2}, d^{2}\right)\right\rangle-k\left(x, u^{1}, d^{1}, u^{2}, d^{2}\right)\right]
\end{aligned}
$$

and for $V \in C\left(\mathbb{R}^{d} ; \mathbb{R}^{m_{1} \times m_{2}}\right)$, let

$$
\begin{aligned}
& M_{-}^{d^{1}, d^{2}}[V](x)=\min _{\bar{d}^{2} \neq d^{2}}\left[V^{d^{1}, \bar{d}^{2}}(x)+c^{2}\left(d^{2}, \bar{d}^{2}\right)\right], \\
& M_{+}^{d^{1}, d^{2}}[V](x)=\max _{\bar{d}^{1} \neq d^{1}}\left[V^{\bar{d}^{1}, d^{2}}(x)-c^{1}\left(d^{1}, \bar{d}^{1}\right)\right], \\
& N\left[V^{d^{1}, d^{2}}\right](x)=\min _{\xi \in K}\left[V^{d^{1}, d^{2}}(x+\xi)+l(\xi)\right] .
\end{aligned}
$$

The HJI upper systems of equations associated to the hybrid differential game are as follows:

$$
\begin{aligned}
& \min \left\{\operatorname { m a x } \left(\lambda V^{d^{1}, d^{2}}+H_{+}^{d^{1}, d^{2}}\left(x, D V^{d^{1}, d^{2}}\right), V^{d^{1}, d^{2}}-M_{-}^{d^{1}, d^{2}}[V],\right.\right. \\
& \left.\left.V^{d^{1}, d^{2}}-N\left[V^{d^{1}, d^{2}}\right]\right), V^{d^{1}, d^{2}}-M_{+}^{d^{1}, d^{2}}[V]\right\}=0 ; \quad\left(d^{1}, d^{2}\right) \in D^{1} \times D^{2}, \\
& \max \left\{\min \left(\lambda V^{d^{1}, d^{2}}+H_{+}^{d^{1}, d^{2}}\left(x, D V^{d^{1}, d^{2}}\right), V^{d^{1}, d^{2}}-M_{+}^{d^{1}, d^{2}}[V]\right),\right. \\
& \left.V^{d^{1}, d^{2}}-M_{-}^{d^{1}, d^{2}}[V], V^{d^{1}, d^{2}}-N\left[V^{d^{1}, d^{2}}\right]\right\}=0 ; \quad\left(d^{1}, d^{2}\right) \in D^{1} \times D^{2} .
\end{aligned}
$$

If we replace $H_{+}^{d^{1}, d^{2}}$ in the above system of equations by $H_{-}^{d^{1}, d^{2}}$, then we obtain the HJI lower system of equations denoted, respectively, by (HJI1-) and (HJI2-). If $V$ satisfies both $(\mathrm{HJI} 1+)$ and $(\mathrm{HJI} 2+)$, then we say that $V$ satisfies $(\mathrm{HJI}+)$ and similarly if it satisfies 
both (HJI-) and (HJI2-), we say that $V$ satisfies (HJI-). In the next section, we show that $V_{-}$satisfies (HJI+) and $V_{+}$satisfies (HJI-) in the viscosity sense.

We say that the Isaacs min-max condition holds if $H_{-}=H_{+}$, Under this condition, the equations $(\mathrm{HJI} 1+)$ and $(\mathrm{HJ} 2+)$, respectively, coincide with (HJI1-) and (HJI2-). We now state the main result of this paper; the proof will be worked out in subsequent sections.

Theorem 1.1. Assume (A1), (A2) and the Isaacs min-max condition. Then $V_{-}=V_{+}$is the unique viscosity solution of $(\mathrm{HJI}+)($ or, $(\mathrm{HJI}-))$ in $B U C\left(\mathbb{R}^{d}, \mathbb{R}^{m_{1} \times m_{2}}\right)$.

\section{Dynamic programming principle}

In this section, we first prove the DPP for differential games with hybrid controls. We first state the results and then the proofs will be given. Throughout this section we assume (A1) and (A2).

Lemma 2.1. For $\left(x, d^{1}, d^{2}\right) \in \mathbb{R}^{d} \times D^{1} \times D^{2}$ and $t>0$,

$$
\begin{aligned}
V_{-}^{d^{1}, d^{2}}(x)= & \inf _{\beta \in \Delta^{d^{2}}} \sup _{\mathscr{C}^{1, d^{1}}}\left[\int _ { 0 } ^ { t } \mathrm { e } ^ { - \lambda s } k \left(y_{x}(s), u^{1}(s), d^{1}(s), \Pi_{1} \beta\left(u^{1}, d^{1}\right)(s),\right.\right. \\
& \left.\Pi_{2} \beta\left(u^{1}, d^{1}\right)(s)\right) \mathrm{d} s-\sum_{\theta_{j}^{1}<t} \mathrm{e}^{-\lambda \theta_{j}^{1}} c^{1}\left(d_{j-1}^{1}, d_{j}^{1}\right) \\
& +\sum_{\left(\Pi_{2} \beta\right)_{1, j}<t} \mathrm{e}^{-\lambda\left(\Pi_{2} \beta\right)_{1, j}} c^{2}\left(\left(\Pi_{2} \beta\right)_{2, j-1},\left(\Pi_{2} \beta\right)_{2, j}\right) \\
& +\sum_{\left(\Pi_{3} \beta\right)_{1, j}<t} \mathrm{e}^{-\lambda\left(\Pi_{3} \beta\right)_{1, j} l\left(\left(\Pi_{3} \beta\right)_{2, j}\right)} \\
& \left.+\mathrm{e}^{-\lambda t} V_{-}^{d^{1}(t), \Pi_{2} \beta(t)}\left(y_{x}(t)\right)\right]
\end{aligned}
$$

Lemma 2.2. For $\left(x, d^{1}, d^{2}\right) \in \mathbb{R}^{d} \times D^{1} \times D^{2}$ and $t>0$,

$$
\begin{aligned}
V_{+}^{d^{1}, d^{2}}(x)= & \sup _{\alpha \in \Gamma^{d^{1}}} \inf _{\mathscr{C}^{2, d^{2}}}\left[\int_{0}^{t} \mathrm{e}^{-\lambda s} k\left(y_{x}(s), \alpha\left(u^{2}, d^{2}, \xi\right)(s), u^{2}(s), d^{2}(s), \xi(s)\right) \mathrm{d} s\right. \\
& -\sum_{\left(\Pi_{2} \alpha\right)_{1, j}<t} \mathrm{e}^{-\lambda\left(\Pi_{2} \alpha\right)_{1, j}} c^{1}\left(\left(\Pi_{2} \alpha\right)_{1, j-1},\left(\Pi_{2} \alpha\right) 1, j\right) \\
& \left.+\sum_{\theta_{j}^{2}<t} \mathrm{e}^{-\lambda \theta_{j}^{2}} c^{2}\left(d_{j-1}^{2}, d_{j}^{2}\right)+\sum_{\tau_{j}<t} \mathrm{e}^{-\lambda \tau_{j}} l\left(\xi_{j}\right)+\mathrm{e}^{-\lambda t} V_{+}^{\Pi_{2} \alpha(t), d^{2}(t)}\left(y_{x}(t)\right)\right] .
\end{aligned}
$$


Lemma 2.3. (i) $M_{+}^{d^{1}, d^{2}}\left[V_{-}\right](x) \leqslant V_{-}^{d^{1}, d^{2}}(x)$.

(ii) $V_{-}^{d^{1}, d^{2}}(x) \leqslant \min \left\{M_{-}^{d^{1}, d^{2}}\left[V_{-}\right](x), N\left[V_{-}^{d^{1}, d^{2}}\right](x)\right\}$.

(iii) Let $\left(x, d^{1}, d^{2}\right)$ be such that strict inequality holds in (i). Let $\bar{\beta} \in \Delta_{0}^{d^{2}}$. Then there exists $t_{0}>0$ such that the following holds:

For each $0 \leqslant t \leqslant t_{0}$, there exists $u^{1, t}(\cdot) \in \mathscr{U}^{1}[0, t]$ such that

$$
\begin{aligned}
V_{-}^{d^{1}, d^{2}}(x)-t^{2} \leqslant \int_{0}^{t} & \mathrm{e}^{-\lambda s} k\left(y_{x}(s), u^{1, t}(s), d^{1}, \bar{\beta}\left(u^{1, t}(\cdot), d^{1}\right)(s) \mathrm{d} s\right. \\
& +\mathrm{e}^{-\lambda t} V_{-}^{d^{1}, d^{2}}\left(y_{x}(t)\right) .
\end{aligned}
$$

(iv) Let $\left(x, d^{1}, d^{2}\right)$ be such that strict inequality holds in (ii). Let $\bar{u}^{1} \in U^{1}$. Then there exists $t_{0}>0$ such that the following holds:

For each $0 \leqslant t \leqslant t_{0}$, there exists $\beta^{t} \in \Delta^{d^{2}}$ with $\left(\Pi_{2} \beta^{t}\left(\bar{u}^{1}, d^{1}\right)\right)_{1,1},\left(\Pi_{3} \beta^{t}\left(\bar{u}^{1}, d^{1}\right)\right)_{1,1}>t_{0}$ such that

$$
V_{-}^{d^{1}, d^{2}}(x)+t^{2} \geqslant \int_{0}^{t} \mathrm{e}^{-\lambda s} k\left(y_{x}(s), \bar{u}^{1}, d^{1}, \beta^{t}\left(\bar{u}^{1}, d^{1}\right)(s)\right) \mathrm{d} s+\mathrm{e}^{-\lambda t} V_{-}^{d^{1}, d^{2}}\left(y_{x}(t)\right) .
$$

Lemma 2.4. (i) $M_{+}^{d^{1}, d^{2}}\left[V_{+}\right](x) \leqslant V_{+}^{d^{1}, d^{2}}(x)$.

(ii) $V_{+}^{d^{1}, d^{2}}(x) \leqslant \min \left\{M_{-}^{d^{1}, d^{2}}\left[V_{+}\right](x), N\left[V_{+}^{d^{1}, d^{2}}\right](x)\right\}$.

(iii) Let $\left(x, d^{1}, d^{2}\right)$ be such that strict inequality holds in (i). Let $\bar{\alpha} \in \Gamma_{0}^{d^{1}}$. Then there exists $t_{0}>0$ such that the following holds:

For each $0 \leqslant t \leqslant t_{0}$, there exists $u^{2, t}(\cdot) \in \mathscr{U}^{2}[0, t]$ such that

$$
\begin{aligned}
V_{+}^{d^{1}, d^{2}}(x)+t^{2} \geqslant & \int_{0}^{t} \mathrm{e}^{-\lambda s} k\left(y_{x}(s), u^{2, t}(s), d^{2}, \bar{\alpha}\left(u^{2, t}, d^{2}, \xi^{\infty}\right)(s)\right) \mathrm{d} s \\
& +\mathrm{e}^{-\lambda t} V_{+}^{d^{1}, d^{2}}\left(y_{x}(t)\right) .
\end{aligned}
$$

(iv) Let $\left(x, d^{1}, d^{2}\right)$ be such that strict inequality holds in (ii). Let $\bar{u}^{2} \in U^{2}$. Then there exists $t_{0}>0$ such that the following holds:

For each $0 \leqslant t \leqslant t_{0}$, there exists $\alpha^{t} \in \Gamma^{d^{1}}$ with $\left(\Pi_{2} \alpha^{t}\left(\bar{u}^{2}, d^{2}, \xi^{\infty}\right)\right)_{1,1}>t_{0}$ such that

$$
V_{+}^{d^{1}, d^{2}}(x)-t^{2} \leqslant \int_{0}^{t} \mathrm{e}^{-\lambda s} k\left(y_{x}(s), \bar{u}^{2}, d^{2}, \alpha^{t}\left(\bar{u}^{2}, d^{2}, \xi^{\infty}\right)(s)\right) \mathrm{d} s+\mathrm{e}^{-\lambda t} V_{+}^{d^{1}, d^{2}}\left(y_{x}(t)\right) .
$$

We prove Lemmas 2.1 and 2.3 and the proofs of Lemmas 2.2 and 2.4 are analogous.

Proof of Lemma 2.1. Let $\left(x, d^{1}, d^{2}\right) \in \mathbb{R}^{d} \times D^{1} \times D^{2}$ and $t>0$. Let us denote the RHS of (2.1) by $W(x)$. Fix $\varepsilon>0$. 
Let $\bar{\beta} \in \Delta^{d^{2}}$ be such that

$$
\begin{aligned}
& W(x) \geqslant \sup _{\mathscr{C}^{1, d^{1}}}\left[\int_{0}^{t} \mathrm{e}^{-\lambda s} k\left(y_{x}(s), u^{1}(s), d^{1}(s), \Pi_{1} \bar{\beta}\left(u^{1}, d^{1}\right)(s), \Pi_{2} \bar{\beta}\left(u^{1}, d^{1}\right)(s)\right) \mathrm{d} s\right. \\
& -\sum_{\theta_{j}^{1}<t} \mathrm{e}^{-\lambda \theta_{j}^{1}} c^{1}\left(d_{j-1}^{1}, d_{j}^{1}\right)+\sum_{\left(\Pi_{2} \bar{\beta}\right)_{1, j}<t} \mathrm{e}^{-\lambda\left(\Pi_{2} \bar{\beta}\right)_{1, j}} c^{2}\left(\left(\Pi_{2} \bar{\beta}\right)_{2, j-1},\left(\Pi_{2} \bar{\beta}\right)_{2, j}\right) \\
& \left.+\sum_{\left(\Pi_{3} \bar{\beta}\right)_{1, j}<t} \mathrm{e}^{-\lambda\left(\Pi_{3} \bar{\beta}\right)_{1, j}} l\left(\left(\Pi_{3} \bar{\beta}\right)_{2, j}\right)+\mathrm{e}^{-\lambda t} V_{-}^{d^{1}(t),\left(\Pi_{2} \bar{\beta}\right)(t)}\left(y_{x}(t)\right)\right]-\varepsilon .
\end{aligned}
$$

By the definition of $V_{-}$, for each $\left(u^{1}(\cdot), d^{1}(\cdot)\right) \in \mathscr{C}^{1, d^{1}}$, there exists $\beta_{u^{1}(\cdot), d^{1}(\cdot)} \in \Delta^{\left(\Pi_{2} \bar{\beta}\right)(t)}$ such that

$$
V_{-}^{d^{1}(t),\left(\Pi_{2} \bar{\beta}\right)(t)}\left(y_{x}(t)\right) \geqslant J_{y_{x}(t)}^{d^{1}(t),\left(\left(\Pi_{2} \bar{\beta}\right)(t)\right.}\left[u^{1}(\cdot), d^{1}(\cdot), \beta_{u^{1}(\cdot), d^{1}(\cdot)}\left(u^{1}(\cdot), d^{1}(\cdot)\right)\right]-\varepsilon .
$$

Define $\delta \in \Delta^{d^{2}}$ by

$$
\delta\left(u^{1}(\cdot), d^{1}(\cdot)\right)(s)= \begin{cases}\bar{\beta}\left(u^{1}(\cdot), d^{1}(\cdot)\right)(s), & s \leqslant t, \\ \beta_{u^{1}(\cdot), d^{1}(\cdot)}\left(u^{1}(\cdot+t), d^{1}(\cdot+t)\right)(s-t), & s>t .\end{cases}
$$

By change of variables, we get

$$
\begin{aligned}
& J_{y_{x}(t)}^{d^{1}(t),\left(\Pi_{2} \bar{\beta}\right)(t)}\left[u^{1}(\cdot+t), d^{1}(\cdot+t), \beta_{u^{1}(\cdot), d^{1}(\cdot)}\left(u^{1}(\cdot+t), d^{1}(\cdot+t)\right)\right] \\
& =\int_{t}^{\infty} \mathrm{e}^{-\lambda \tau} k\left(y_{x}(\tau), u^{1}(\tau), d^{1}(\tau),\left(\Pi_{1} \delta\right)\left(u^{1}, d^{1}\right)(\tau),\left(\Pi_{2} \delta\right)\left(u^{1}, d^{1}\right)(\tau)\right) \mathrm{d} \tau \\
& \quad-\sum_{\theta_{j}^{1}>t} \mathrm{e}^{-\lambda \theta_{j}^{1}} c^{1}\left(d_{j-1}^{1}, d_{j}^{1}\right) \\
& \quad+\sum_{\left(\Pi_{2} \delta\right)_{1, j}>t} \mathrm{e}^{-\lambda\left(\Pi_{2} \delta\right)_{1, j}} c^{2}\left(\left(\Pi_{2} \delta\right)_{2, j-1},\left(\Pi_{2} \delta\right)_{2, j}\right) \\
& \quad+\sum_{\left(\Pi_{3} \delta\right)_{1, j}>t} \mathrm{e}^{-\lambda\left(\Pi_{3} \delta\right)_{1, j} l\left(\left(\Pi_{3} \delta\right)_{2, j}\right) .}
\end{aligned}
$$

Therefore

$$
V_{-}^{d^{1}(t),\left(\Pi_{2} \bar{\beta}\right)(t)}\left(y_{x}(t)\right) \geqslant(*)-\varepsilon,
$$

where $(*)$ denotes the RHS of the above equation. This implies that

$$
W(x) \geqslant J_{x}^{d^{1}, d^{2}}\left[u^{1}(\cdot), d^{1}(\cdot), \delta\left(u^{1}, d^{1}\right)(\cdot)\right]-2 \varepsilon .
$$


This holds for all $\left(u^{1}(\cdot), d^{1}(\cdot)\right) \in \mathscr{C}^{1, d^{1}}$ and hence

$$
W(x) \geqslant V_{-}^{d^{1}, d^{2}}(x)-2 \varepsilon \text {. }
$$

Since $\varepsilon>0$ is arbitrary, we get $W(x) \geqslant V_{-}^{d^{1}, d^{2}}(x)$.

We now prove the other way inequality. Fix $\beta \in \Delta^{d^{2}}$ and $\varepsilon>0$. Choose $\left(\bar{u}^{1}(\cdot), \bar{d}^{1}(\cdot)\right) \in$ $\mathscr{C}^{1, d^{1}}$ such that

$$
\begin{aligned}
W(x) \leqslant & \int_{0}^{t} \mathrm{e}^{-\lambda s} k\left(y_{x}(s), \bar{u}^{1}(s), \bar{d}^{1}(s), \beta\left(\bar{u}^{1}, \bar{d}^{1}\right)(s)\right) \mathrm{d} s \\
& -\sum_{\theta_{j}^{1}<t} \mathrm{e}^{-\lambda \theta_{j}^{1}} c^{1}\left(d_{j-1}^{1}, d_{j}^{1}\right) \\
& +\sum_{\left(\Pi_{2} \beta\right)_{1, j}<t} \mathrm{e}^{-\lambda\left(\Pi_{2} \beta\right)_{1, j}} c^{2}\left(\left(\Pi_{2} \beta\right)_{2, j-1},\left(\Pi_{2} \beta\right)_{2, j}\right) \\
& +\sum_{\left(\Pi_{3} \beta\right)_{1, j}<t} \mathrm{e}^{-\lambda\left(\Pi_{3} \beta\right)_{1, j}} l\left(\left(\Pi_{3} \beta\right)_{2, j}\right) \\
& +\mathrm{e}^{-\lambda t} V_{-}^{\bar{d}^{1}(t),\left(\Pi_{2} \beta\right)(t)}\left(y_{x}(t)\right)+\varepsilon .
\end{aligned}
$$

Now for each $u^{1}(\cdot)$, define $\tilde{u}^{1}(\cdot)$ by

$$
\tilde{u}^{1}(s)= \begin{cases}\bar{u}^{1}(s), & s \leqslant t, \\ u^{1}(s-t), & s>t .\end{cases}
$$

Similarly, for each $d^{1}(\cdot)$, we define $\tilde{d}^{1}(\cdot)$. Let

$$
\hat{\beta}\left(u^{1}(\cdot), d^{1}(\cdot)\right)(s)=\beta\left(\tilde{u}^{1}(\cdot), \tilde{d}^{1}(\cdot)\right)(s+t) .
$$

By the definition of $V_{-}$, we can choose $\left(u^{1}(\cdot), d^{1}(\cdot)\right) \in \mathscr{C}^{1, \bar{d}^{1}(t)}$ such that

$$
\begin{aligned}
& V_{-}^{\bar{d}^{1}(t),\left(\Pi_{2} \beta\right)(t)}\left(y_{x}(t)\right) \\
& \left.\leqslant J_{y_{x}(t)}^{\bar{d}^{1}(t),\left(\Pi_{2} \beta\right)(t)}\left[u^{1}(\cdot+t), d^{1}(\cdot+t), \hat{\beta}\left(u^{1}, d^{1}\right)(\cdot+t)\right)\right]+\varepsilon \mathrm{e}^{\lambda t} .
\end{aligned}
$$

Now, combining (2.2) and (2.3), we get

$$
\begin{aligned}
& W(x) \leqslant \int_{0}^{t} \mathrm{e}^{-\lambda s} k\left(y_{x}(s), \bar{u}^{1}(s), \bar{d}^{1}(s), \beta\left(\bar{u}^{1}, \bar{d}^{1}\right)(s)\right) \mathrm{d} s-\sum_{\theta_{j}^{1}<t} \mathrm{e}^{-\lambda \theta_{j}^{1}} c^{1}\left(d_{j-1}^{1}, d_{j}^{1}\right) \\
& +\sum_{\left(\Pi_{2} \beta\right)_{1, j}<t} \mathrm{e}^{-\lambda \Pi_{2} \beta_{1, j}} c^{2}\left(\left(\Pi_{2} \beta\right)_{2, j-1},\left(\Pi_{2} \beta\right)_{2, j}\right) \\
& +\sum_{\Pi_{3} \beta_{1, j}<t} \mathrm{e}^{-\lambda\left(\Pi_{3} \beta\right)_{1, j}} l\left(\left(\Pi_{3} \beta\right)_{2, j}\right)+\mathrm{e}^{-\lambda t} J_{y_{x}(t)}^{\bar{d}^{1}(t),\left(\Pi_{2} \beta\right)(t)}\left[u^{1}(\cdot), d^{1}(\cdot), \hat{\beta}\left(u^{1}(\cdot), d^{1}(\cdot)\right)\right]+2 \varepsilon .
\end{aligned}
$$

By change of variables, it follows that

$$
\left.W(x) \leqslant J_{x}^{d^{1}, d^{2}}\left[\tilde{u}^{1}(\cdot), \tilde{d}^{1}(\cdot), \beta\left(\tilde{u}^{1}, \tilde{d}^{1}\right)(\cdot)\right)\right]+2 \varepsilon .
$$


This holds for any $\beta \in \Delta^{d^{2}}$ and hence

$$
W(x) \leqslant V_{-}^{d^{1}, d^{2}}(x)+2 \varepsilon .
$$

The proof is now complete, since $\varepsilon$ is arbitrary.

Proof of Lemma 2.3. We first prove (i) and (ii). By the definition of $V_{-}$, for any $\bar{d}^{2} \neq d^{2}$ $V_{-}^{d^{1}, d^{2}}(x) \leqslant V_{-}^{d^{1}, \bar{d}^{2}}(x)+c^{2}\left(d^{2}, \bar{d}^{2}\right)$.

From this we get

$$
V_{-}^{d^{1}, d^{2}}(x) \leqslant M_{-}^{d^{1}, d^{2}}\left[V_{-}\right](x) .
$$

The inequality

$$
V_{-}^{d^{1}, d^{2}}(x) \geqslant M_{+}^{d^{1}, d^{2}}\left[V_{-}\right](x)
$$

can be proved in a similar fashion.

Clearly for any $\xi \in K$ and $\beta \in \Delta^{d^{2}}$

$$
V_{-}^{d^{1}, d^{2}}(x) \leqslant \sup _{\mathscr{C}^{1, d^{1}}} J_{x+\xi}^{d^{1}, d^{2}}\left[u^{1}(\cdot), d^{1}(\cdot), \beta\left(u^{1}, d^{1}\right)(\cdot)\right]+l(\xi) .
$$

First take infimum over $\beta \in \Delta^{d^{2}}$ and then over $\xi \in K$, we obtain

$$
V_{-}^{d^{1}, d^{2}}(x) \leqslant N\left[V_{-}^{d^{1}, d^{2}}\right](x) .
$$

We now turn to the proof of (iii). By Lemma 2.1, for each $t \geqslant 0$, there exists $\left(u^{1, t}(\cdot), d^{1, t}\right.$ $(\cdot)) \in \mathscr{C}^{1, d^{1}}$ such that

$$
\begin{aligned}
V_{-}^{d^{1}, d^{2}}(x)-t^{2} \leqslant & \int_{0}^{t} \mathrm{e}^{-\lambda s} k\left(y_{x}(s), u^{1, t}(s), d^{1, t}(s), \bar{\beta}\left(u^{1, t}, d^{1, t}\right)(s)\right) \mathrm{d} s \\
& -\sum_{\theta_{j}^{1, t}<t} \mathrm{e}^{-\lambda \theta_{j}^{1, t}} c^{1}\left(d_{j-1}^{1, t}, d_{j}^{1, t}\right)+\mathrm{e}^{-\lambda t} V_{-}^{d^{1, t}(t),\left(\Pi_{2} \bar{\beta}\right)(t)}\left(y_{x}(t)\right) .
\end{aligned}
$$

It is enough to show that, for some $t_{0}>0, \theta_{1}^{1, t} \geqslant t$ for all $0 \leqslant t \leqslant t_{0}$. If this does not happen, then there would exist a sequence $t_{n} \downarrow 0$ such that $\theta_{1}^{1, t_{n}}<t_{n}$ for all $n$. This would imply that

$$
\begin{aligned}
V_{-}^{d^{1}, d^{2}}(x)-\left(\theta_{1}^{1, t_{n}}\right)^{2} \leqslant & \int_{0}^{\theta_{1}^{1, t_{n}}} \mathrm{e}^{-\lambda s} k\left(y_{x}(s), u^{1, t_{n}}(s), d^{1, t_{n}}(s), \bar{\beta}\left(u^{1, t_{n}}, d^{1, t_{n}}\right)(s)\right) \mathrm{d} s \\
& -\mathrm{e}^{-\lambda \theta_{1}^{1, t_{n}}} c^{1}\left(d^{1}, d_{1}^{1, t_{n}}\right)+\mathrm{e}^{-\lambda \theta_{1}^{1, t_{n}} V_{-}^{d_{1}^{1, t_{n}}, d^{2}}}\left(y_{x}\left(\theta_{1}^{1, t_{n}}\right)\right) .
\end{aligned}
$$

We may assume that for all $n, d_{1}^{1, t_{n}}=\bar{d}^{1} \neq d^{1}$. Now by letting $n \rightarrow \infty$ in the above inequality, we get

$$
\begin{aligned}
V_{-}^{d^{1}, d^{2}}(x) & \leqslant-c^{1}\left(d^{1}, \bar{d}^{1}\right)+V_{-}^{\bar{d}^{1}, d^{2}}(x) \\
& \leqslant M_{+}^{d^{1}, d^{2}}\left[V_{-}\right](x) .
\end{aligned}
$$


This contradicts the hypothesis that strict inequality holds in (i) and the proof of (iii) is now complete.

We next prove (iv). By Lemma 2.1, for each $t>0$, there exists $\beta^{t} \in \Delta^{d^{2}}$ such that

$$
\begin{aligned}
V_{-}^{d^{1}, d^{2}}(x)+t^{2} \geqslant & \int_{0}^{t} \mathrm{e}^{-\lambda s} k\left(y_{x}(s), \bar{u}^{1}, d^{1}, \beta^{t}\left(\bar{u}^{1}, d^{1}\right)(s)\right) \mathrm{d} s \\
& +\mathrm{e}^{-\lambda t} V_{-}^{d^{1},\left(\Pi_{2} \beta^{t}\right)(t)}\left(y_{x}(t)\right) \\
& +\sum_{\left(\Pi_{2} \beta^{t}\right)_{1, j}<t} \mathrm{e}^{-\lambda \theta_{j}^{2, t} c^{2}\left(d_{j-1}^{2, t}, d_{j}^{2, t}\right)} \\
& +\sum_{\left(\Pi_{3} \beta^{t}\right)_{1, j}<t} \mathrm{e}^{-\lambda \tau_{j}^{t}} l\left(\xi_{j}^{t}\right) .
\end{aligned}
$$

It is enough to show that, for some $t_{0}>0, \min \left(\theta_{1}^{2, t}, \tau_{1}^{t}\right) \geqslant t$ for all $0 \leqslant t \leqslant t_{0}$. If this were not true, then (without any loss of generality) there would be a sequence $t_{n} \downarrow 0$ and two cases to consider. In the first case, $\theta_{1}^{2, t_{n}} \leqslant \min \left(t_{n}, \tau_{1}^{t_{n}}\right)$ whereas in the second case $\tau_{1}^{t_{n}} \leqslant \min \left(t_{n}, \theta_{1}^{2, t_{n}}\right)$. By dropping to a subsequence if necessary and proceeding as in the proof of (iii), we get $V_{-}^{d^{1}, d^{2}}(x) \geqslant M_{-}^{d^{1}, d^{2}}\left[V_{-}\right](x)$ and $V_{-}^{d^{1}, d^{2}}(x) \geqslant N\left[V_{-}^{d^{1}, d^{2}}\right](x)$ in case 1 and case 2 respectively. This contradicts our hypothesis that strict inequality holds in (ii) and the proof is now complete.

Remark 2.5. From the proofs it is clear that, instead of the term $t^{2}$ in the statement of Lemmas 2.3 and 2.4, we can take any modulus $\rho(t)$.

By properly modifying the arguments in the proof of Lemma 1.11, Chapter VIII in [1], we can prove the next lemma. We omit the details.

Lemma 2.6. Let $\eta>0, \phi \in C^{1}\left(\mathbb{R}^{d}\right)$ and $\left(x, d^{1}, d^{2}\right) \in \mathbb{R}^{d} \times D^{1} \times D^{2}$ be such that

$$
\lambda \phi(x)+H_{+}^{d^{1}, d^{2}}(x, D \phi(x))=\eta .
$$

Then there exists $\bar{\beta} \in \Delta_{0}^{d^{2}}$ such that for all $\left(u^{1}(\cdot), d^{1}(\cdot)\right) \in \mathscr{C}^{1, d^{1}}$ and $t$ small enough,

$$
\begin{aligned}
& \int_{0}^{t} \mathrm{e}^{-\lambda s}\left[\lambda \phi\left(y_{x}(s)\right)-\left\langle D \phi\left(y_{x}(s)\right), f\left(y_{x}(s), u^{1}(s), d^{1}(s), \bar{\beta}\left(u^{1}, d^{1}\right)(s)\right)\right\rangle\right. \\
& \left.\quad-k\left(y_{x}(s), u^{1}(s), d^{1}(s), \bar{\beta}\left(u^{1}, d^{1}\right)(s)\right)\right] \mathrm{d} s \geqslant \frac{\eta}{2} t .
\end{aligned}
$$

We are now ready to prove the fact that $V_{-}$(resp., $\left.V_{+}\right)$is a viscosity solution of (HJ+) (resp., (HJI-)).

Theorem 2.7. The lower value function $V_{-}$is a viscosity solution of $(\mathrm{HJI}+)$ and the upper value function $V_{+}$is a viscosity solution of (HJI-). 
Proof. We prove that $V_{-}$is a viscosity solution of (HJI+). The other part can be proved in an analogous manner.

We first prove that $V_{-}$is a subsolution of $(\mathrm{HJI} 1+)$. Let $\left(x, d^{1}, d^{2}\right) \in \mathbb{R}^{d} \times D^{1} \times D^{2}$ and $\phi \in C^{1}\left(\mathbb{R}^{d}\right)$ be such that $V_{-}^{d^{1}, d^{2}}-\phi$ has a local maximum at $x$. Without any loss of generality, we may assume that $V_{-}^{d^{1}, d^{2}}(x)=\phi(x)$. If $V_{-}^{d^{1}, d^{2}}(x)=M_{+}^{d^{1}, d^{2}}\left[V_{-}\right](x)$, then we are done. Assume that $V_{-}^{d^{1}, d^{2}}(x)>M_{+}^{d^{1}, d^{2}}\left[V_{-}\right](x)$. It suffices to show that

$$
\lambda \phi(x)+H_{+}^{d^{1}, d^{2}}(x, D \phi(x))=: r \leqslant 0 .
$$

If possible, let $r>0$. By Lemma 2.6, there exists $\bar{\beta} \in \Delta_{0}^{d^{2}}$ such that for all $\left(u^{1}(\cdot), d^{1}(\cdot)\right) \in$ $\mathscr{C}^{1, d^{1}}$ and $t$ small enough

$$
\begin{aligned}
\frac{r}{2} t \leqslant & \int_{0}^{t} \mathrm{e}^{-\lambda s}\left[\lambda \phi\left(y_{x}(s)\right)-\left\langle D \phi\left(y_{x}(s)\right), f\left(y_{x}(s), u^{1}(s), d^{1}(s), \bar{\beta}\left(u^{1}, d^{1}\right)(s)\right)\right\rangle\right. \\
& \left.-k\left(y_{x}(s), u^{1}(s), d^{1}(s), \bar{\beta}\left(u^{1}, d^{1}\right)(s)\right)\right] \mathrm{d} s \\
= & \left.\phi(x)-\mathrm{e}^{-\lambda t} \phi\left(y_{x}(t)\right)-\int_{0}^{t} \mathrm{e}^{-\lambda s} k\left(y_{x}(s), u^{1}(s), d^{1}(s), \bar{\beta}\left(u^{1}, d^{1}\right)(s)\right)\right] \mathrm{d} s \\
\leqslant & V_{-}^{d^{1}, d^{2}}(x)-\mathrm{e}^{-\lambda t} V_{-}^{d^{1}, d^{2}}\left(y_{x}(t)\right) \\
& \left.-\int_{0}^{t} \mathrm{e}^{-\lambda s} k\left(y_{x}(s), u^{1}(s), d^{1}(s), \bar{\beta}\left(u^{1}, d^{1}\right)(s)\right)\right] \mathrm{d} s .
\end{aligned}
$$

By Lemma 2.3(iii), for $t$ small enough, there exits $u^{1, t}(\cdot) \in \mathscr{U}^{1}[0, t]$ with

$$
V_{-}^{d^{1}, d^{2}}(x)-t^{2} \leqslant \int_{0}^{t} \mathrm{e}^{-\lambda s} k\left(y_{x}(s), u^{1, t}(s), d^{1}, \bar{\beta}\left(u^{1, t}(\cdot), d^{1}\right)(s) \mathrm{d} s+\mathrm{e}^{-\lambda t} V_{-}^{d^{1}, d^{2}}\left(y_{x}(t)\right)\right. \text {. }
$$

Therefore, for $t$ small enough, we obtain $\frac{r}{2} t \leqslant t^{2}$. This contradiction proves the fact that $V_{-}$is a subsolution of $(\mathrm{HJI} 1+)$.

To prove that $V_{-}$is a supersolution of (HJI1+), let $x$ be a local minimum of $V_{-}^{d^{1}, d^{2}}-\phi$. Without any loss of generality, we may assume that $V_{-}^{d^{1}, d^{2}}(x)=\phi(x)$. If $V_{-}^{d^{1}, d^{2}}(x)=$ $M_{-}^{d^{1}, d^{2}}\left[V_{-}\right](x)$ or $V_{-}^{d^{1}, d^{2}}(x)=N\left[V_{-}^{d^{1}, d^{2}}\right](x)$, then we are done. Assume that $V_{-}^{d^{1}, d^{2}}(x)<\min$ $\left(M_{-}^{d^{1}, d^{2}}\left[V_{-}\right](x), N\left[V_{-}^{d^{1}, d^{2}}\right](x)\right)$. In this case, we need to show that

$$
\lambda \phi(x)+H_{+}^{d^{1}, d^{2}}(x, D \phi(x))=: \hat{r} \geqslant 0 .
$$

If possible, let $\hat{r}<0$. Then

$$
\lambda \phi(x)-\left\langle D \phi(x), f\left(x, \bar{u}^{1}, d^{1}, u^{2}, d^{2}\right)\right\rangle-k\left(\left(x, \bar{u}^{1}, d^{1}, u^{2}, d^{2}\right) \leqslant \frac{\hat{r}}{2}\right.
$$

for some $\bar{u}^{1} \in U^{1}$ and all $u^{2} \in U^{2}$. This implies that, for all $\beta \in \Delta_{0}^{d^{2}}$ and $s$ small enough

$$
\begin{aligned}
& \lambda \phi\left(y_{x}(s)\right)-\left\langle D \phi\left(y_{x}(s)\right), f\left(y_{x}(s), \bar{u}^{1}, d^{1}, \beta\left(\bar{u}^{1}, d^{1}\right)(s)\right)\right\rangle \\
& \quad-k\left(y_{x}(s), \bar{u}^{1}, d^{1}, \beta\left(\bar{u}^{1}, d^{1}\right)(s)\right) \leqslant \frac{\hat{r}}{4} .
\end{aligned}
$$


Multiplying throughout by $\mathrm{e}^{-\lambda s}$ and integrating from 0 to $t$, we get

$$
\begin{aligned}
& \frac{\hat{r}}{4} t \geqslant \phi(x)-\mathrm{e}^{-\lambda t} \phi\left(y_{x}(t)\right)-\int_{0}^{t} \mathrm{e}^{-\lambda s} k\left(y_{x}(s), \bar{u}^{1}, d^{1}, \beta\left(\bar{u}^{1}, d^{1}\right)(s)\right) \mathrm{d} s \\
& \quad \geqslant V_{-}^{d^{1}, d^{2}}(x)-\mathrm{e}^{-\lambda t} V_{-}^{d^{1}, d^{2}}\left(y_{x}(t)\right)-\int_{0}^{t} \mathrm{e}^{-\lambda s} k\left(y_{x}(s), \bar{u}^{1}, d^{1}, \beta\left(\bar{u}^{1}, d^{1}\right)(s)\right) \mathrm{d} s .
\end{aligned}
$$

Now by Lemma 2.3(iv), for $t$ small enough, there exists $\beta^{t} \in \Delta_{0}^{d^{2}}$ such that

$$
V_{-}^{d^{1}, d^{2}}(x)+t^{2} \geqslant \int_{0}^{t} \mathrm{e}^{-\lambda s} k\left(y_{x}(s), \bar{u}^{1}, d^{1}, \beta^{t}\left(\bar{u}^{1}, d^{1}\right)(s)\right) \mathrm{d} s+\mathrm{e}^{-\lambda t} V_{-}^{d^{1}, d^{2}}\left(y_{x}(t)\right) .
$$

Therefore, for $t$ small, we obtain $-t^{2} \leqslant \frac{\hat{r}}{4} t$. This is a contradiction and proves the fact that $V_{-}$is a supersolution of $(\mathrm{HJI} 1+)$. In a similar fashion, we can show that $V_{-}$is a viscosity solution of $(\mathrm{HJ} 2+)$. Hence $V_{-}$is a viscosity solution of $(\mathrm{HJI}+)$.

\section{Proof of the main result}

In this section we prove the main theorem of the paper, namely uniqueness. First, we state and prove two lemmas needed in the proof of uniqueness. The proof of first lemma is same as in [5], but we give the proof for the sake of completeness.

Lemma 3.1. Assume (A2). Let $w$ be uniformly continuous.

$$
\text { If } w^{d^{1}, d^{2}}\left(y_{0}\right)=N\left[w^{d^{1}, d^{2}}\right]\left(y_{0}\right)=w^{d^{1}, d^{2}}\left(y_{0}+\xi_{0}\right)+l\left(\xi_{0}\right),
$$

then there exists $\sigma>0$ (which depends only on $w$ ) such that for all $y \in \bar{B}\left(y_{0}+\xi_{0}, \sigma\right)$,

$$
w^{d^{1}, d^{2}}(y)<N\left[w^{d^{1}, d^{2}}\right](y) .
$$

Proof. Let

$$
w^{d^{1}, d^{2}}\left(y_{0}\right)=N\left[w^{d^{1}, d^{2}}\right]\left(y_{0}\right)=w^{d^{1}, d^{2}}\left(y_{0}+\xi_{0}\right)+l\left(\xi_{0}\right) .
$$

Then, for every $\xi_{1} \in K$

$$
\begin{aligned}
& w^{d^{1}, d^{2}}\left(y_{0}+\xi_{0}+\xi_{1}\right)+l\left(\xi_{1}\right)-w^{d^{1}, d^{2}}\left(y_{0}+\xi_{0}\right) \\
& \quad=w^{d^{1}, d^{2}}\left(y_{0}+\xi_{0}+\xi_{1}\right)+l\left(\xi_{1}\right)-w^{d^{1}, d^{2}}\left(y_{0}\right)+l\left(\xi_{0}\right) \\
& \quad \geqslant-l\left(\xi_{0}+\xi_{1}\right)+l\left(\xi_{0}\right)+l\left(\xi_{1}\right) .
\end{aligned}
$$

Hence

$$
\begin{aligned}
N\left[w^{d^{1}, d^{2}}\left(y_{0}+\xi_{0}\right)\right]-w^{d^{1}, d^{2}}\left(y_{0}+\xi_{0}\right) & \geqslant \inf _{\xi_{1}}\left[l\left(\xi_{0}\right)+l\left(\xi_{1}\right)-l\left(\xi_{0}+\xi_{1}\right)\right] \\
& =\bar{l}>0 .
\end{aligned}
$$


By using uniform continuity of $w^{d^{1}, d^{2}}$ and $N\left[w^{d^{1}, d^{2}}\right]$ we get a $\sigma$ such that for all $y \in$ $\bar{B}\left(y_{0}+\xi_{0}, \sigma\right)$,

$$
w^{d^{1}, d^{2}}(y)<N\left[w^{d^{1}, d^{2}}\right](y) .
$$

Lemma 3.2. Assume (A1) and (A2).

(i) Any supersolution $w$ of $(\mathrm{HJI} 1+)$ satisfies $w^{d^{1}, d^{2}} \geqslant M_{+}^{d^{1}, d^{2}}[w]$ for all $d^{1}, d^{2}$.

(ii) Any subsolution $w$ of (HJI2+) satisfies $w^{d^{1}, d^{2}} \leqslant \min \left(M_{-}^{d^{1}, d^{2}}[w], N\left[w^{d^{1}, d^{2}}\right]\right)$ for all $d^{1}, d^{2}$.

Proof. Let $w$ be a supersolution of (HJI1+). If possible, let

$$
w^{d^{1}, d^{2}}\left(x_{0}\right)<M_{+}^{d^{1}, d^{2}}[w]\left(x_{0}\right) .
$$

By continuity, the above holds for all $x$ in an open ball $B$ around $x_{0}$. By Lemma 1.8(d), p. 30 in [1], there exists $y_{0} \in B$ and a smooth map $\phi$ such that $w^{d^{1}, d^{2}}-\phi$ has local minimum at $y_{0}$. Since $w$ is a supersolution of (HJI1+), this will lead to a contradiction

$$
w^{d^{1}, d^{2}}\left(y_{0}\right) \geqslant M_{+}^{d^{1}, d^{2}}[w]\left(y_{0}\right) .
$$

This proves (i). The proof of (ii) is similar.

Next is the proof of uniqueness theorem.

Theorem 3.3. Assume (A1) and (A2). Let $v$ and $w \in B U C\left(\mathbb{R}^{d} ; \mathbb{R}^{m_{1} \times m_{2}}\right)$ be viscosity solutions of $(\mathrm{HJI}+)($ or $(\mathrm{HJI}-))$. Then, $v=w$.

Proof. We prove the uniqueness for $(\mathrm{HJI}+)$. The result for $(\mathrm{HJI}-)$ is similar.

Let $v$ and $w$ be viscosity solutions of (HJI+). We prove $v^{d^{1}, d^{2}} \leqslant w^{d^{1}, d^{2}}$ for all $d^{1}, d^{2}$. In a similar fashion we can prove that $w^{d^{1}, d^{2}} \leqslant v^{d^{1}, d^{2}}$ for all $d^{1}, d^{2}$.

For $\left(d^{1}, d^{2}\right) \in D^{1} \times D^{2}$, define $\Phi^{d^{1}, d^{2}}: \mathbb{R}^{d} \times \mathbb{R}^{d} \rightarrow \mathbb{R}$ by

$$
\Phi^{d^{1}, d^{2}}(x, y)=v^{d^{1}, d^{2}}(x)-w^{d^{1}, d^{2}}(y)-\frac{|x-y|^{2}}{2 \varepsilon}-\kappa\left[\langle x\rangle^{\bar{m}}+\langle y\rangle^{\bar{m}}\right],
$$

where $\bar{m} \in(0,1) \cap\left(0, \frac{\lambda}{\|f\|_{\infty}}\right)$ is fixed, $\kappa, \varepsilon \in(0,1)$ are parameters, and $\langle x\rangle^{\bar{m}}=\left(1+\|x\|^{2}\right)^{\bar{m} / 2}$.

Note that $x \rightarrow\langle x\rangle^{\bar{m}}$ is Lipschitz continuous with Lipschitz constant 1 .

Let $M_{0}=\sup _{\xi \in K}|\xi|$. We first fix $\kappa>0$. Let $\left(x_{\varepsilon}, y_{\varepsilon}\right)$ and $\left(d_{\varepsilon}^{1}, d_{\varepsilon}^{2}\right)$ be such that

$$
\Phi^{d_{\varepsilon}^{1}, d_{\varepsilon}^{2}}\left(x_{\varepsilon}, y_{\varepsilon}\right)=\sup _{x, y} \max _{d^{1}, d^{2}} \Phi^{d^{1}, d^{2}}(x, y) .
$$

By Lemma 2.3, we have

$$
w^{d_{\varepsilon}^{1}, d_{\varepsilon}^{2}}\left(y_{\varepsilon}\right) \leqslant N\left[w^{d_{\varepsilon}^{1}, d_{\varepsilon}^{2}}\right]\left(y_{\varepsilon}\right)
$$




$$
\begin{aligned}
& w^{d_{\varepsilon}^{1}, d_{\varepsilon}^{2}}\left(y_{\varepsilon}\right) \leqslant M_{-}^{d_{\varepsilon}^{1}, d_{\varepsilon}^{2}}[w]\left(y_{\varepsilon}\right), \\
& v^{d_{\varepsilon}^{1}, d_{\varepsilon}^{2}}\left(x_{\varepsilon}\right) \geqslant M_{+}^{d_{\varepsilon}^{1}, d_{\varepsilon}^{2}}[v]\left(x_{\varepsilon}\right) .
\end{aligned}
$$

If we have strict inequality in all the above three equations (that is, 3.1-3.3), then by the definition of viscosity sub and super solution we will have

$$
\begin{aligned}
& \lambda v^{d_{\varepsilon}^{1}, d_{\varepsilon}^{2}}\left(x_{\varepsilon}\right)+H_{+}^{d_{\varepsilon}^{1}, d_{\varepsilon}^{2}}\left(x_{\varepsilon}, \frac{x_{\varepsilon}-y_{\varepsilon}}{\varepsilon}+\kappa \bar{m}\left\langle x_{\varepsilon}\right\rangle^{\bar{m}-2} x_{\varepsilon}\right) \leqslant 0, \\
& \lambda w^{d_{\varepsilon}^{1}, d_{\varepsilon}^{2}}\left(y_{\varepsilon}\right)+H_{+}^{d_{\varepsilon}^{1}, d_{\varepsilon}^{2}}\left(y_{\varepsilon}, \frac{x_{\varepsilon}-y_{\varepsilon}}{\varepsilon}-\kappa \bar{m}\left\langle y_{\varepsilon}\right\rangle^{\bar{m}-2} y_{\varepsilon}\right) \geqslant 0 .
\end{aligned}
$$

In this case we can proceed by the usual comparison principle method. Thus we first show that for some auxiliary function at the maximum point strict inequality occurs in (3.1), (3.2) and (3.3). We deal these cases one by one. For, let there be equality in (3.1). i.e.,

$$
w^{d_{\varepsilon}^{1}, d_{\varepsilon}^{2}}\left(y_{\varepsilon}\right)=N\left[w^{d_{\varepsilon}^{1}, d_{\varepsilon}^{2}}\right]\left(y_{\varepsilon}\right) .
$$

By the definition of $N$, since $\xi$ varies in the compact set, let $\xi_{\varepsilon}$ be such that

$$
w^{d_{\varepsilon}^{1}, d_{\varepsilon}^{2}}\left(y_{\varepsilon}\right)=N\left[w^{d_{\varepsilon}^{1}, d_{\varepsilon}^{2}}\right]\left(y_{\varepsilon}\right)=w^{d_{\varepsilon}^{1}, d_{\varepsilon}^{2}}\left(y_{\varepsilon}+\xi_{\varepsilon}\right)+l\left(\xi_{\varepsilon}\right) .
$$

Then,

$$
\begin{aligned}
\Phi^{d_{\varepsilon}^{1}, d_{\varepsilon}^{2}}\left(x_{\varepsilon}+\xi_{\varepsilon}, y_{\varepsilon}+\xi_{\varepsilon}\right)= & v^{d_{\varepsilon}^{1}, d_{\varepsilon}^{2}}\left(x_{\varepsilon}+\xi_{\varepsilon}\right)-w^{d_{\varepsilon}^{1}, d_{\varepsilon}^{2}}\left(y_{\varepsilon}+\xi_{\varepsilon}\right)-\frac{\left|x_{\varepsilon}-y_{\varepsilon}\right|^{2}}{2 \varepsilon} \\
& -\kappa\left[\left\langle x_{\varepsilon}+\xi_{\varepsilon}\right\rangle^{\bar{m}}+\left\langle y_{\varepsilon}+\xi_{\varepsilon}\right\rangle^{\bar{m}}\right] \\
\geqslant & v^{d_{\varepsilon}^{1}, d_{\varepsilon}^{2}}\left(x_{\varepsilon}\right)-w^{d_{\varepsilon}^{1}, d_{\varepsilon}^{2}}\left(y_{\varepsilon}\right)-\frac{\left|x_{\varepsilon}-y_{\varepsilon}\right|^{2}}{2 \varepsilon} \\
& -\kappa\left[\left\langle x_{\varepsilon}\right\rangle^{\bar{m}}+\left\langle y_{\varepsilon}\right\rangle^{\bar{m}}\right]-2 \kappa\left|\xi_{\varepsilon}\right| \\
= & \Phi^{d_{\varepsilon}^{1}, d_{\varepsilon}^{2}}\left(x_{\varepsilon}, y_{\varepsilon}\right)-2 \kappa\left|\xi_{\varepsilon}\right| \\
\geqslant & \Phi^{d_{\varepsilon}^{1}, d_{\varepsilon}^{2}}\left(x_{\varepsilon}, y_{\varepsilon}\right)-2 \kappa M_{0} .
\end{aligned}
$$

Hence we have

$$
\Phi^{d_{\varepsilon}^{1}, d_{\varepsilon}^{2}}\left(x_{\varepsilon}, y_{\varepsilon}\right)-\Phi^{d_{\varepsilon}^{1}, d_{\varepsilon}^{2}}\left(x_{\varepsilon}+\xi_{\varepsilon}, y_{\varepsilon}+\xi_{\varepsilon}\right) \leqslant 2 \kappa M_{0} .
$$

We will be using this difference to define the new auxiliary function.

Observe that $\left|x_{\varepsilon}\right|,\left|y_{\varepsilon}\right|,\left|\xi_{\varepsilon}\right|$ are bounded hence without any loss of generality we may assume that

$$
\left(x_{\varepsilon}, y_{\varepsilon}\right) \rightarrow\left(x_{0}, y_{0}\right) \text { and } \xi_{\varepsilon} \rightarrow \xi_{0} .
$$

We define the new auxiliary function $\Psi$ by

$$
\Psi^{d^{1}, d^{2}}(x, y)=\Phi^{d^{1}, d^{2}}(x, y)+2 \kappa M_{0} \eta\left(\frac{x-x_{0}-\xi_{0}}{\sigma}, \frac{y-y_{0}-\xi_{0}}{\sigma}\right),
$$


where $\sigma=\sigma(w)$ is the constant coming from Lemma 3.1 and $\eta: \mathbb{R}^{d} \times \mathbb{R}^{d} \rightarrow \mathbb{R}$ is a smooth function with the following properties:

1. $\operatorname{supp}(\eta) \subset B((0,0), 1)$,

2. $0 \leqslant \eta \leqslant 1$,

3. $\eta(0,0)=1$ and $\eta<1$ if $(x, y) \neq(0,0)$,

4. $|D \eta| \leqslant 1$.

Now by the definition of $\Psi$

$$
\Psi^{d_{\varepsilon}^{1}, d_{\varepsilon}^{2}}\left(x_{0}+\xi_{0}, y_{0}+\xi_{0}\right)=\Phi^{d_{\varepsilon}^{1}, d_{\varepsilon}^{2}}\left(x_{0}+\xi_{0}, y_{0}+\xi_{0}\right)+2 \kappa M_{0} \geqslant \Phi^{d_{\varepsilon}^{1}, d_{\varepsilon}^{2}}\left(x_{0}, y_{0}\right)
$$

and

$$
\Psi^{d_{\varepsilon}^{1}, d_{\varepsilon}^{2}}(x, y)=\Phi^{d_{\varepsilon}^{1}, d_{\varepsilon}^{2}}(x, y) \quad \text { if }\left|x-x_{0}\right|^{2}+\left|y-y_{0}\right|^{2} \geqslant \sigma^{2} .
$$

Hence $\Psi^{d_{\varepsilon}^{1}}, d_{\varepsilon}^{2}$ attains its maximum in the $\sigma$ ball around $\left(x_{0}+\xi_{0}, y_{0}+\xi_{0}\right)$ at say $\left(\hat{x}_{\varepsilon}, \hat{y}_{\varepsilon}\right)$. By Lemma 3.1 we now know that

$$
w^{d_{\varepsilon}^{1}, d_{\varepsilon}^{2}}\left(\hat{y}_{\varepsilon}\right)<N\left[w^{d_{\varepsilon}^{1}, d_{\varepsilon}^{2}}\right]\left(\hat{y}_{\varepsilon}\right) .
$$

If strict inequality holds in (3.2) at $\hat{y}_{\varepsilon}$ and in (3.3) at $\hat{x}_{\varepsilon}$ then $w^{d_{\varepsilon}^{1}, d_{\varepsilon}^{2}}$ and $v^{d_{\varepsilon}^{1}}, d_{\varepsilon}^{2}$ satisfy (3.5) and (3.4), respectively. In this case we can proceed by usual method.

If not let there be equality in (3.2). That is

$$
w^{d_{\varepsilon}^{1}, d_{\varepsilon}^{2}}\left(\hat{y}_{\varepsilon}\right)=M_{-}^{d_{\varepsilon}^{1}, d_{\varepsilon}^{2}}[w]\left(\hat{y}_{\varepsilon}\right) .
$$

By the definition of $M_{-}^{d_{\varepsilon}^{1}, d_{\varepsilon}^{2}}$, there exists $d_{\varepsilon_{1}}^{2} \in D^{2}$ such that

$$
w^{d_{\varepsilon}^{1}, d_{\varepsilon}^{2}}\left(\hat{y}_{\varepsilon}\right)=w^{d_{\varepsilon}^{1}, d_{\varepsilon_{1}}^{2}}\left(\hat{y}_{\varepsilon}\right)+c^{2}\left(d_{\varepsilon}^{2}, d_{\varepsilon_{1}}^{2}\right) .
$$

We know that

$$
v^{d_{\varepsilon}^{1}, d_{\varepsilon}^{2}}\left(\hat{x}_{\varepsilon}\right) \leqslant v^{d_{\varepsilon}^{1}, d_{\varepsilon_{1}}^{2}}\left(\hat{x}_{\varepsilon}\right)+c^{2}\left(d_{\varepsilon}^{2}, d_{\varepsilon_{1}}^{2}\right) .
$$

Hence

$$
\begin{aligned}
\Psi^{d_{\varepsilon}^{1}, d_{\varepsilon}^{2}}\left(\hat{x}_{\varepsilon}, \hat{y}_{\varepsilon}\right) \leqslant & v^{d_{\varepsilon}^{1}, d_{\varepsilon_{1}}^{2}}\left(\hat{x}_{\varepsilon}\right)+c^{2}\left(d_{\varepsilon}^{2}, d_{\varepsilon_{1}}^{2}\right)-w^{d_{\varepsilon}^{1}, d_{\varepsilon_{1}}^{2}}\left(\hat{y}_{\varepsilon}\right)-c^{2}\left(d_{\varepsilon}^{2}, d_{\varepsilon_{1}}^{2}\right) \\
& -\frac{\left|\hat{x}_{\varepsilon}-\hat{y}_{\varepsilon}\right|^{2}}{2 \varepsilon}-\kappa\left[\left\langle\hat{x}_{\varepsilon}\right\rangle^{\bar{m}}+\left\langle\hat{y}_{\varepsilon}\right\rangle^{\bar{m}}\right]+2 \kappa M_{0} \eta\left(\hat{x}_{\varepsilon}, \hat{y}_{\varepsilon}\right) \\
\leqslant & \Psi^{d_{\varepsilon}^{1}, d_{\varepsilon_{1}}^{2}}\left(\hat{x}_{\varepsilon}, \hat{y}_{\varepsilon}\right) .
\end{aligned}
$$

But by the definition of $\left(d_{\varepsilon}^{1}, d_{\varepsilon}^{2}\right)$,

$$
\Psi^{d_{\varepsilon}^{1}, d_{\varepsilon}^{2}}\left(\hat{x}_{\varepsilon}, \hat{y}_{\varepsilon}\right) \geqslant \Psi^{d_{\varepsilon}^{1}, d_{\varepsilon_{1}}^{2}}\left(\hat{x}_{\varepsilon}, \hat{y}_{\varepsilon}\right) .
$$

Hence

$$
\Psi^{d_{\varepsilon}^{1}, d_{\varepsilon}^{2}}\left(\hat{x}_{\varepsilon}, \hat{y}_{\varepsilon}\right)=\Psi^{d_{\varepsilon}^{1}, d_{\varepsilon_{1}}^{2}}\left(\hat{x}_{\varepsilon}, \hat{y}_{\varepsilon}\right) .
$$


Now if the strict inequality holds in $w^{d_{\varepsilon}^{1}, d_{\varepsilon_{1}}^{2}}\left(\hat{y}_{\varepsilon}\right) \leqslant M_{-}^{d_{\varepsilon}^{1}, d_{\varepsilon_{1}}^{2}}[w]\left(\hat{y}_{\varepsilon}\right)$ we proceed to check if strict inequality holds in (3.3) else we repeat the above argument and get $d_{\varepsilon_{2}}^{2} \in D^{2}$ such that,

$$
\begin{aligned}
w^{d_{\varepsilon}^{1}, d_{\varepsilon_{1}}^{2}}\left(\hat{y}_{\varepsilon}\right) & =w^{d_{\varepsilon}^{1}, d_{\varepsilon_{2}}^{2}}\left(\hat{y}_{\varepsilon}\right)+c^{2}\left(d_{\varepsilon_{1}}^{2}, d_{\varepsilon_{2}}^{2}\right), \\
w^{d_{\varepsilon}^{1}, d_{\varepsilon_{2}}^{2}}\left(\hat{y}_{\varepsilon}\right) & =w^{d_{\varepsilon}^{1}, d_{\varepsilon_{1}}^{2}}\left(\hat{y}_{\varepsilon}\right)-c^{2}\left(d_{\varepsilon_{1}}^{2}, d_{\varepsilon_{2}}^{2}\right) \\
& \geqslant w^{d_{\varepsilon}^{1}, d_{\varepsilon_{1}}^{2}}\left(\hat{y}_{\varepsilon}\right)-c_{0}^{2} \\
& \geqslant w^{d_{\varepsilon}^{1}, d_{\varepsilon}^{2}}\left(\hat{y}_{\varepsilon}\right)-2 c_{0}^{2}
\end{aligned}
$$

proceeding in similar fashion after finitely many steps, boundedness of $w$ will be contradicted and thus for some $d_{\varepsilon_{k}}^{2} \in D^{2}$ strict inequality will hold at $\hat{y}_{\varepsilon}$ in (3.2).

Now if strict inequality holds at $\hat{x}_{\varepsilon}$ in (3.3), we can proceed by usual comparison method else let us assume that equality holds in (3.3).

As before then we have that

$$
v^{d_{\varepsilon}^{1}, d_{\varepsilon_{k}}^{2}}\left(\hat{x}_{\varepsilon}\right)=M_{+}^{d_{\varepsilon}^{1}, d_{\varepsilon_{k}}^{2}}[v]\left(\hat{x}_{\varepsilon}\right)
$$

and by the definition of $M_{+}$, there exists $d_{\varepsilon_{1}}^{1}$ such that

$$
v^{d_{\varepsilon}^{1}, d_{\varepsilon_{k}}^{2}}\left(\hat{x}_{\varepsilon}\right)=v^{d_{\varepsilon_{1}}^{1}, d_{\varepsilon_{k}}^{2}}\left(\hat{x}_{\varepsilon}\right)-c^{1}\left(d_{\varepsilon_{1}}^{1}, d_{\varepsilon}^{1}\right) .
$$

This case can be handled exactly similarly to the case

$$
w^{d_{\varepsilon}^{1}, d_{\varepsilon}^{2}}\left(\hat{y}_{\varepsilon}\right)=M_{-}^{d_{\varepsilon}^{1}, d_{\varepsilon}^{2}}[w]\left(\hat{y}_{\varepsilon}\right) .
$$

Thus for some $d^{1} \varepsilon_{j} \in D^{1}$ strict inequality will hold at $\hat{x}_{\varepsilon}$ in (3.3).

By Lemma 2.3 we have

$$
v^{d_{\varepsilon}^{1}, d_{\varepsilon_{k}}^{2}}\left(\hat{y}_{\varepsilon}\right) \geqslant v^{d_{\varepsilon_{1}}^{1}, d_{\varepsilon_{k}}^{2}}\left(\hat{y}_{\varepsilon}\right)-c^{1}\left(d_{\varepsilon_{1}}^{1}, d_{\varepsilon}^{1}\right)
$$

Hence

$$
\begin{aligned}
\Psi^{d_{\varepsilon}^{1}, d_{\varepsilon_{k}}^{2}}\left(\hat{x}_{\varepsilon}, \hat{y}_{\varepsilon}\right) \leqslant & v^{d_{\varepsilon_{1}}^{1}, d_{\varepsilon_{k}}^{2}}\left(\hat{x}_{\varepsilon}\right)-c^{1}\left(d_{\varepsilon_{1}}^{1}, d_{\varepsilon}^{1}\right)-w^{d_{\varepsilon_{1}}^{1}, d_{\varepsilon_{k}}^{2}}\left(\hat{y}_{\varepsilon}\right)+c^{1}\left(d_{\varepsilon_{1}}^{1}, d_{\varepsilon}^{1}\right) \\
& -\frac{\left|\hat{x}_{\varepsilon}-\hat{y}_{\varepsilon}\right|^{2}}{2 \varepsilon}-\kappa\left[\left\langle\hat{x}_{\varepsilon}\right\rangle^{\bar{m}}+\left\langle\hat{y}_{\varepsilon}\right\rangle^{\bar{m}}\right]+2 \kappa M_{0} \eta\left(\hat{x}_{\varepsilon}, \hat{y}_{\varepsilon}\right) \\
\leqslant & \Psi^{d_{\varepsilon_{1}}^{1}, d_{\varepsilon_{k}}^{2}}\left(\hat{x}_{\varepsilon}, \hat{y}_{\varepsilon}\right) .
\end{aligned}
$$

Hence

$$
\Psi^{d_{\varepsilon}^{1}, d_{\varepsilon_{k}}^{2}}\left(\hat{x}_{\varepsilon}, \hat{y}_{\varepsilon}\right)=\Psi^{d_{\varepsilon_{1}}^{1}, d_{\varepsilon_{k}}^{2}}\left(\hat{x}_{\varepsilon}, \hat{y}_{\varepsilon}\right)
$$

Now if the strict inequality holds in, $v^{d_{\varepsilon_{1}}^{1}, d_{\varepsilon_{k}}^{2}}\left(\hat{x}_{\varepsilon}\right) \leqslant M_{+}^{d_{\varepsilon_{1}}^{1}, d_{\varepsilon_{k}}^{2}}[v]\left(\hat{x}_{\varepsilon}\right)$ then we are done. If there is equality, then we repeat the above argument by replacing index $\varepsilon$ by $\varepsilon_{1}$ and get an $d_{\varepsilon_{2}}^{1} \in D^{1}$ such that,

$$
v^{d_{\varepsilon_{1}}^{1}, d_{\varepsilon_{k}}^{2}}\left(\hat{x}_{\varepsilon}\right)=v^{d_{\varepsilon_{2}}^{1}, d_{\varepsilon_{k}}^{2}}\left(\hat{x}_{\varepsilon}\right)+c^{1}\left(d_{\varepsilon_{1}}^{1}, d_{\varepsilon_{2}}^{1}\right) \text {. }
$$


Since $c^{1}\left(d_{\varepsilon}^{1}, d_{\varepsilon_{1}}^{1}\right), c^{1}\left(d_{\varepsilon_{1}}^{1}, d_{\varepsilon_{2}}^{1}\right)>0$, the possibility $\varepsilon_{1}=\varepsilon$ is ruled out by (3.8). Thus, for some $d_{\varepsilon_{j}}^{1} \in D^{1}$ strict inequality will hold at $\hat{x}_{\varepsilon}$ in (3.3).

Thus we have at $\left(\hat{x}_{\varepsilon}, \hat{y}_{\varepsilon}\right)$, the maximizer of the auxiliary function $\Psi^{d_{\varepsilon_{j}}^{1}, d_{\varepsilon_{k}}^{2}}$ strict inequality holds in all the three inequalities (3.1)-(3.3).

Now we define test functions $\phi_{1}$ and $\phi_{2}$ as follows:

$$
\begin{aligned}
\phi_{1}(x)= & w^{d_{\varepsilon_{j}}^{1}, d_{\varepsilon_{k}}^{2}}\left(\hat{y}_{\varepsilon}\right)+\frac{\left|x-\hat{y}_{\varepsilon}\right|^{2}}{2 \varepsilon}+\kappa\left[\langle x\rangle^{\bar{m}}+\left\langle\hat{y}_{\varepsilon}\right\rangle^{\bar{m}}\right] \\
& -2 \kappa M_{0} \eta\left(\frac{x-x_{0}-\xi_{0}}{\sigma}, \frac{\hat{y}_{\varepsilon}-y_{0}-\xi_{0}}{\sigma}\right), \\
\phi_{2}(y)= & v^{d_{\varepsilon_{j}}^{1}, d_{\varepsilon_{k}}^{2}}\left(\hat{x}_{\varepsilon}\right)-\frac{\left|\hat{x}_{\varepsilon}-y\right|^{2}}{2 \varepsilon}-\kappa\left[\left\langle\hat{x}_{\varepsilon}\right\rangle^{\bar{m}}+\langle y\rangle^{\bar{m}}\right] \\
& +2 \kappa M_{0} \eta\left(\frac{\hat{x}_{\varepsilon}-x_{0}-\xi_{0}}{\sigma}, \frac{y-y_{0}-\xi_{0}}{\sigma}\right) .
\end{aligned}
$$

Observe that

$$
\begin{aligned}
& D \phi_{1}\left(\hat{x}_{\varepsilon}\right)=\frac{\hat{x}_{\varepsilon}-\hat{y}_{\varepsilon}}{\varepsilon}+\kappa \bar{m}\left\langle\hat{x}_{\varepsilon}\right\rangle^{m-2} \hat{x}_{\varepsilon}-\frac{2 \kappa M_{0}}{\sigma} D \eta\left(\frac{\hat{x}_{\varepsilon}-x_{0}-\xi_{0}}{\sigma}, \frac{\hat{y}_{\varepsilon}-y_{0}-\xi_{0}}{\sigma}\right), \\
& D \phi_{2}\left(\hat{y}_{\varepsilon}\right)=\frac{\hat{x}_{\varepsilon}-\hat{y}_{\varepsilon}}{\varepsilon}-\kappa \bar{m}\left\langle\hat{y}_{\varepsilon}\right\rangle^{m-2} \hat{y}_{\varepsilon}+\frac{2 \kappa M_{0}}{\sigma} D \eta\left(\frac{\hat{x}_{\varepsilon}-x_{0}-\xi_{0}}{\sigma}, \frac{\hat{y}_{\varepsilon}-y_{0}-\xi_{0}}{\sigma}\right) .
\end{aligned}
$$

Note that $v^{d_{\varepsilon_{j}}^{1}, d_{\varepsilon_{k}}^{2}}-\phi_{1}$ attains its maximum at $\hat{x}_{\varepsilon}$ and $w^{d_{\varepsilon_{j}}^{1}, d_{\varepsilon_{k}}^{2}}-\phi_{2}$ attains its minimum at $\hat{y}_{\varepsilon}$. Hence

$$
\begin{aligned}
\lambda\left[v^{d_{\varepsilon_{j}}^{1}, d_{\varepsilon_{k}}^{2}}\left(\hat{x}_{\varepsilon}\right)-w^{d_{\varepsilon_{j}}^{1}, d_{\varepsilon_{k}}^{2}}\left(\hat{y}_{\varepsilon}\right)\right] \leqslant & H_{+}^{d_{\varepsilon_{j}}^{1}, d_{\varepsilon_{k}}^{2}}\left(\hat{y}_{\varepsilon}, D \phi_{2}\left(\hat{y}_{\varepsilon}\right)\right)-H_{+}^{d_{\varepsilon_{j}}^{1}, d_{\varepsilon_{k}}^{2}}\left(\hat{x}_{\varepsilon}, D \phi_{1}\left(\hat{x}_{\varepsilon}\right)\right) \\
\leqslant & L\left|\hat{x}_{\varepsilon}-\hat{y}_{\varepsilon}\right|\left(1+\left|\frac{\hat{x}_{\varepsilon}-\hat{y}_{\varepsilon}}{\varepsilon}\right|\right) \\
& +\|f\|_{\infty}\left[\kappa \bar{m}\left(\left\langle\hat{x}_{\varepsilon}\right\rangle^{\bar{m}-1}+\left\langle\hat{y}_{\varepsilon}\right\rangle^{\bar{m}-1}\right)+\frac{4 \kappa M_{0}}{\sigma}\right] .
\end{aligned}
$$

Note that we have used $\left|\hat{y}_{\varepsilon}\right| \leqslant\left(1+\left|\hat{y}_{\varepsilon}\right|^{2}\right)^{1 / 2}=\left\langle\hat{y}_{\varepsilon}\right\rangle$ to get the above inequality. Now as $\bar{m}-1<0$ it follows that

$$
\begin{aligned}
v^{d_{\varepsilon_{j}}^{1}, d_{\varepsilon_{k}}^{2}}\left(\hat{x}_{\varepsilon}\right)-w^{d_{\varepsilon_{j}}^{1}, d_{\varepsilon_{k}}^{2}}\left(\hat{y}_{\varepsilon}\right) & \leqslant \frac{L}{\lambda}\left|\hat{x}_{\varepsilon}-\hat{y}_{\varepsilon}\right|+\frac{L}{\lambda} \frac{\left|\hat{x}_{\varepsilon}-\hat{y}_{\varepsilon}\right|^{2}}{\varepsilon}+\frac{2\|f\|_{\infty} \kappa \bar{m}}{\lambda}+\frac{4\|f\|_{\infty} \kappa M_{0}}{\sigma \lambda} \\
& \leqslant \frac{2\|f\|_{\infty} \kappa \bar{m}}{\lambda}+\frac{4\|f\|_{\infty} \kappa M_{0}}{\sigma \lambda}+o(1) ; \quad \text { as } \varepsilon \downarrow 0 .
\end{aligned}
$$


For any $x \in \mathbb{R}^{d}$ and $\left(d^{1}, d^{2}\right) \in D^{1} \times D^{2}$,

$$
\begin{aligned}
v^{d^{1}, d^{2}}(x)-w^{d^{1}, d^{2}}(x)-2 \kappa\langle x\rangle^{\bar{m}} & \leqslant \Psi^{d^{1}, d^{2}}(x, x) \\
\leqslant & \Psi^{d_{\varepsilon_{j}}^{1}, d_{\varepsilon_{k}}^{2}}\left(\hat{x}_{\varepsilon}, \hat{y}_{\varepsilon}\right) \\
\leqslant & v^{d_{\varepsilon_{j}}^{1}, d_{\varepsilon_{k}}^{2}}\left(\hat{x}_{\varepsilon}\right)-w^{d_{\varepsilon_{j}}^{1}, d_{\varepsilon_{k}}^{2}}\left(\hat{y}_{\varepsilon}\right)+2 \kappa M_{0} \\
\leqslant & \frac{2\|f\|_{\infty} \kappa \bar{m}}{\lambda}+\frac{4\|f\|_{\infty} \kappa M_{0}}{\sigma \lambda} \\
& +2 \kappa M_{0}+o(1) ; \quad \text { as } \varepsilon \downarrow 0 .
\end{aligned}
$$

By letting $\varepsilon \downarrow 0$ and then $\kappa \downarrow 0$, we get

$$
v^{d^{1}, d^{2}}(x)-w^{d^{1}, d^{2}}(x) \leqslant 0 .
$$

This completes the proof of uniqueness for $(\mathrm{HJI}+)$.

Under the Isaacs min-max condition, $(\mathrm{HJI}-)$ and $(\mathrm{HJI}+)$ coincide. Hence, the main result (Theorem 1.1) follows from Theorems 2.7 and 3.3.

\section{Conclusions}

We have studied two-person zero-sum differential games with hybrid controls. The minimizing player uses continuous, switching, and impulse controls whereas the maximizing player uses continuous and switching controls. The DPP for lower and upper value functions is proved and using this we have established the existence and uniqueness of the value under Isaacs min-max condition.

Similar result had been obtained by Yong [5] under two additional assumptions:

(Y1) cheaper switching cost condition

$$
\min _{\bar{d}^{2} \neq d^{2}} c^{2}\left(d^{2}, \bar{d}^{2}\right)=: c_{0}^{2}<l_{0}=\inf _{\xi \in K} l(\xi),
$$

(Y2) nonzero loop switching cost condition.

For any loop $\left\{\left(d_{i}^{1}, d_{i}^{2}\right)\right\}_{i=1}^{j} \subset D^{1} \times D^{2}$, with the property that

$$
\begin{aligned}
& j \leqslant m_{1} m_{2}, \quad d_{j+1}^{1}=d_{1}^{1}, \quad d_{j+1}^{2}=d_{1}^{2} ; \\
& \text { either } d_{i+1}^{1}=d_{i}^{1}, \quad \text { or } \quad d_{i+1}^{2}=d_{i}^{2} \quad \forall 1 \leqslant i \leqslant j,
\end{aligned}
$$

it holds that

$$
\sum_{i=1}^{j} c^{1}\left(d_{i}^{1}, d_{i+1}^{1}\right)-\sum_{i=1}^{j} c^{2}\left(d_{i}^{2}, d_{i+1}^{2}\right) \neq 0 .
$$

Hence, we prove the existence and uniqueness of value without the above two conditions (Y1) and (Y2). Moreover, we state and prove the explicit formulation and proof of DPP for hybrid differential games which is not done in [5]. 


\section{Acknowledgements}

The authors wish to thank M.K. Ghosh for suggesting the problem and for several useful discussions. They also thank Mythily Ramaswamy for carefully reading the manuscript and suggesting several modifications.

\section{References}

[1] M. Bardi, I. Capuzzo-Dolcetta, Optimal Control and Viscosity Solutions of Hamilton-Jacobi-Bellman Equations, Birkhauser, Basel, 1997.

[2] L.C. Evans, P.E. Souganidis, Differential games and representation formulas for Hamilton-Jacobi equations, Indiana Univ. Math. J. 33 (1984) 773-797.

[3] J. Yong, Differential games with switching strategies, J. Math. Anal. Appl. 145 (1990) 455-469.

[4] J. Yong, A zero-sum differential game in a finite duration with switching strategies, SIAM J. Control Optim. 28 (1990) 1234-1250.

[5] J. Yong, Zero-sum differential games involving impulse controls, Appl. Math. Optim. 29 (1994) $243-261$. 